\title{
AN INTEGRAL CYCLE MODE CONTROL OF SERIES RESONANT CONVERTER
}

\author{
G. B. Joung, C. T. Rim and G. H. Cho
}

Dept. of Electrical Engineering of Korea Advanced Institute of Science and Technology

\section{ABSTRACT}

A new control method of series resonant converter(SRC) of which the device switching instants are always synchronized to the zero crossing points of the resonant current is proposed. Output voltage is controlled by proper selection of the switch modes by varying the duty ratio of powering mode and free resonant mode. Each switch mode is analyzed and the dc transfer function is obtained. It is also shown that the simulation results are good coinsided with the practical case. The SRC with the proposed control scheme shows many advantages such as low current switching stress, low switching loss, low electromagnetic interference(EMI), high input power factor and wide control range. Some of these characteristics are verified though the experiment.

\section{INTRODUCTION}

Resonant converters are becoming widely used throughout the industry and aerospace applications due to their numerous merits[1-9]. Conventional series resonant converters(SRC's) can be classified into two types according to the control method. One is the frequency domain control scheme and the other is the phase domain control scheme. In the frequency domain control scheme, the output voltage is controlled by the ratio of the switching frequency and the circuit resonant frequency $[1-5,7,8]$. On the other hand, in the phase domain control scheme, it is achieved by the phase difference between two inverters where the switching frequency of each converter is fixed to the resonant frequency[9].

In the frequency domain control scheme, the output voltage depends largely on the load condition as well as the switching frequency, which makes it very difficult to control the system properly[7]. Especially the control range of the output voltage becomes narrow for light load operation and the device current switching stress becomes severe when the switching frequency deviates from the resonant frequency [6]. Phase domain control scheme is more advantageous than the frequency domain control scheme in terms of the load independency. However, this scheme also undergoes severe current switching stress as phase difference becomes large[4].

It is well known that the SRC operates optimally when the switching frequency exactly coincides with the resonant frequency. The output voltage can not be controlled, however, so far as the frequency and phase are fixed. Therefore a time domain control method is suggested in this paper to adjust the output voltage still satisfying its optimum operating conditions.

This proposed control scheme has inherent advantages such as zero current switching stress, low switching loss, low EMI and wide control range. Switch modes are analyzed for a SRC with MOSFET and a practical control scheme is suggested and verified through the experiment.

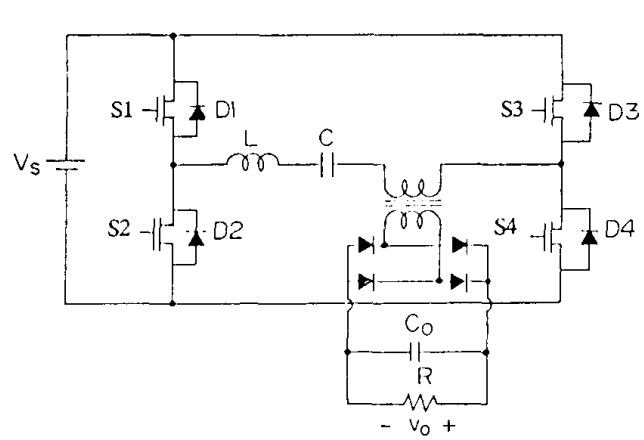

Fig. 1 Power circuit topology of SRC.

II. OPERATING MODES AND CONTROL

\section{METHOD OF SRC}

\section{A. Operating Modes}

The basic power circuit topology of the SRC is shown in Fig. 1. The power circuit has four switch modes as shown in Fig. 2, which are named as powering mode, free resonant mode, regeneration mode and discontinuous mode, respectively. The above modes are classified according to the direction of power flow. Switch on/off control always occurs in synchronization with the current zero crossing points.

Denoting the circuit quantities as input voltage $V_{s}$, output voltage $v_{o}$, inductor current $i_{L}$, capacitor voltage $v_{c}$, and the resonant tank circuit and load parameters $\omega_{r}, Z$ and $Q$ where

$$
\begin{aligned}
& \omega_{r}=\frac{1}{\sqrt{L C}} \\
& Z=\sqrt{L / C} \\
& Q=\omega_{r} L / R
\end{aligned}
$$

the description of each mode is as follows:

(A) Powering mode: $\$ 1, \$ 4$ and $\$ 2, \$ 3$ pairs are turned on and off alternately in synchronization with the current zero crossings. The applied voltage $v$ across the tank circuit and the resonant current $i_{L}$ are in phase as shown in Fig. 3(a). During this mode, the source power is delivered to the $L C$ tank including the load. 


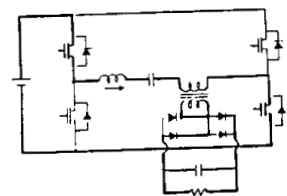

(a)

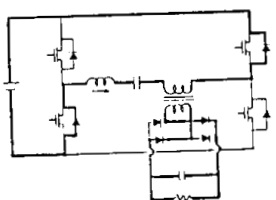

(c)

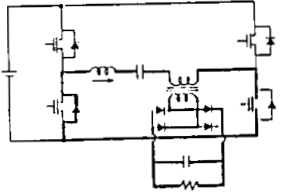

(b)

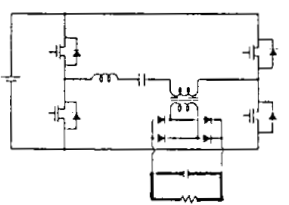

(d)
Fig. 2 Four modes of SRC operation. (a) powering mode (b) free resonant mode (c) regeneration mode (d) discontinuous mode.

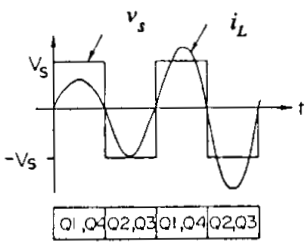

(a)

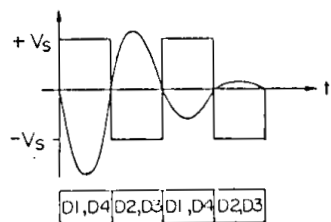

(c)

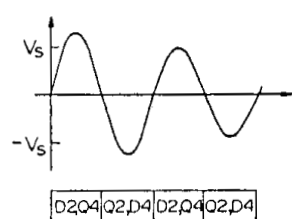

(b)

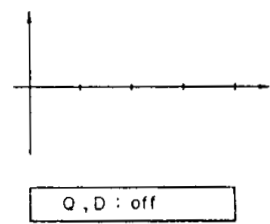

(d)
Fig. 3 Tank circuit waveforms of $i$ and $v$ for each mode

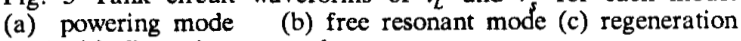
mode (c) discontinuous mode.

(B) Free resonant mode: D2,S4 and S2,D4 pairs(or equivalently $\mathrm{D} 1, \mathrm{S3}$ and $\mathrm{S} 1, \mathrm{D} 3$ pairs) are turned on and off alternately. In this mode, power is not supplied to the tank circuit while the sinusoidal resonant current free-runs and gradually decreases as shown in Fig. 3(b). In this mode, the $L C$ tank energy is delivered to the load.

(C) Regeneration mode: All of the forced commutation
switches(S1-S4) are turned off. Instead, the D1,D4 and D2,D3 pairs are alternately turned on and off in synchronization with the resonant current. During this mode, the applied voltage across the tank circuit and resonant current are out of phase, so the resonant current decreases faster than the current of the free resonant mode. As shown in Fig. 3(c), the $L C$ tank energy is returned to the source while transferring part of the energy to the load.

(D) Discontinuous mode: As the previous free resonant or regeneration mode goes on, the tank resonant current decreases and it can reach to zero as shown in Fig. 3(d) where there is no energy stored in the $L C$ tank.

From these characteristics, the operation modes are summarized in Table. $I$.

\section{B. Mode Control Methods}

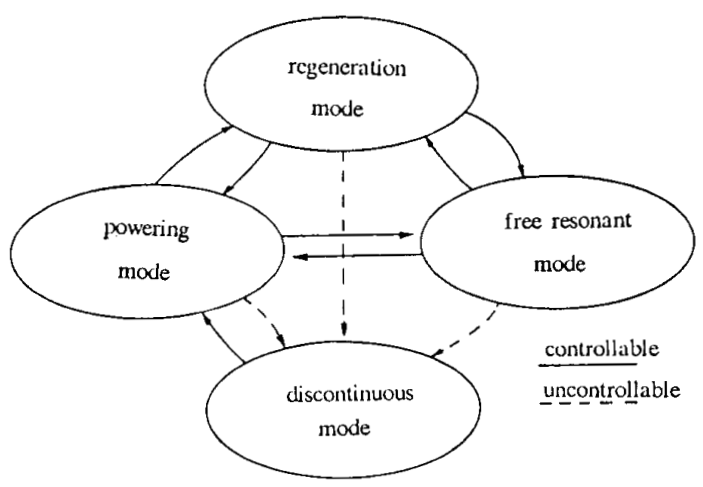

Fig. 4 The flow diagram of the time domain control scheme.

The output voltage of SRC can be controlled by moderate selection of the four modes. Mode selection is allowed only at current zero crossing points, thus the mode interval length should be an integer multiple of half cycle of the resonant frequency. In this sense, it is name as integral cycle mode control scheme. The diagram of the time domain control scheme is shown in Fig .4. In this diagram, powering, free resonant and regeneration modes are selectable by the switches S1-S4. Thus these modes are controllable, however, discontinuous mode is not controllable by the switches since this mode occurs independent of the external switches.

Powering mode increases the output voltage whereas the free resonant mode and the regeneration mode decrease it. Thus the output can be controlled by combining the powering mode and one of the free resonant and regeneration modes properly. Comparing the two passive modes, the regeneration mode deteriorates the input power factor, thus it is undesirable to include this mode in the control scheme.

Table. I. Characteristics of the each mode.

\begin{tabular}{|c|c|c|c|c|c|}
\hline $\begin{array}{c}\text { tank circuit } \\
\text { modes variables }\end{array}$ & voltage & current & $\begin{array}{c}\text { input power } \\
P_{i}\end{array}$ & $\begin{array}{c}\text { output power } \\
P_{o}\end{array}$ & $P_{i}-P_{o}$ \\
\hline powering mode & $\pm V_{s}$ & $\pm\left|i_{L}(t)\right|$ & $V_{s}\left|i_{L}(t)\right|$ & $V_{o}\left|i_{L}(t)\right|$ & $\left(V_{s}-V_{o}\right)\left|i_{L}(t)\right|$ \\
\hline free resonant mode & 0 & $\pm\left|i_{L}(t)\right|$ & 0 & $V_{o}\left|i_{L}(t)\right|$ & $-V_{o}\left|i_{L}(t)\right|$ \\
\hline regeneration mode & $\mp V_{s}$ & $\pm\left|i_{L}(t)\right|$ & $-V_{s}\left|i_{L}(t)\right|$ & $V_{o}\left|i_{L}(t)\right|$ & $-\left(V_{s}+V_{o}\right)\left|i_{L}(t)\right|$ \\
\hline discontinuous mode & 0 & 0 & 0 & 0 & 0 \\
\hline
\end{tabular}




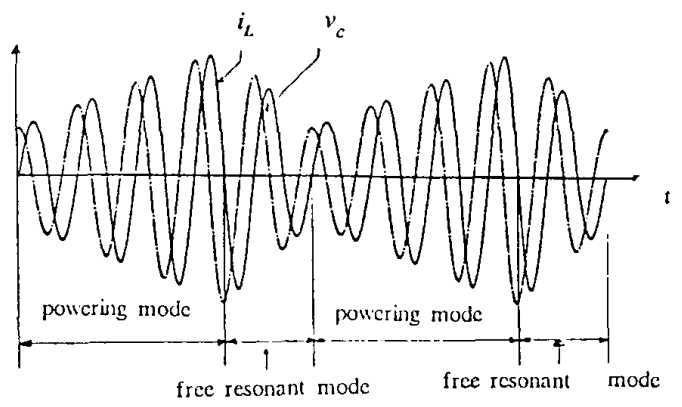

Fig. 5 Typical inductor current and capacitor voltage waveforms in the proposed integral cycle mode control.

Therefore the powering mode and free resonant mode with (or without) discontinuous mode are used for the output voltage control in this paper. Fig. 5 briefly illustrates the waveforms of the proposed control scheme. In this control scheme, the output voltage is regulated by the duty ratio of the powering mode and free resonant mode with (or without) discontinuous mode. As the duty ratio increases, the output voltage is also increased.

\section{ANALYSIS OF THE PROPOSED CONTROL SCHEME}

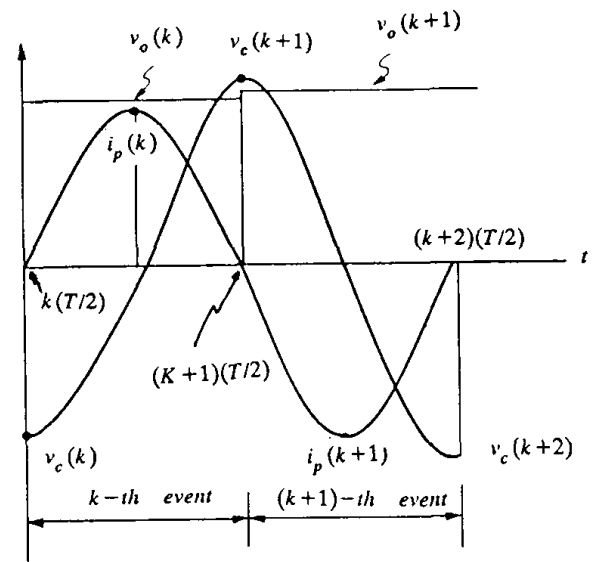

Fig. 6 Representation of the events.

The equations to analyze the resonant operation is shown in Appendix. I for respective four modes. However, it takes long time for exact analysis and also it makes very difficult to understand the time domain control characteristics.

For simplicity, the operation modes of SRC can be approximately analyzed by low ripple approximation method[4] because the output filter capacitor $\left(C_{0}\right)$ is sufficiently larger than the tank circuit capacitor $(C)$ in the practical case. In order to apply the low ripple approximation method, all variables during the $k-t h$ resonant period that is, $k T / 2 \leqslant$ $t<(k+1) T / 2$ are denoted by index $k$. As indicated in Fig. 6 , $v_{o}(k)$ is the output voltage, $v_{c}(k)$ is the tank capacitor voltage at the switching instant and $i_{p}(k)$ is peak current during the $k-t h$ event.

Because the magnitude of the capacitor voltage is related to the stored energy of the capacitor and the magnitude of the inductor current is also related to the stored energy of the inductor, we can change the variables $v_{c}(k), i_{p}(k)$ into new variables $v_{c}^{*}(k), i_{p}^{*}(k)$, where,

$$
\begin{aligned}
& \nu_{c}^{*}(k)=\left|v_{c}(k)\right| \\
& i_{p}^{*}(k)=\left|i_{p}(k)\right|
\end{aligned}
$$

and assuming that $C_{o}>>C$, then the discrete state equations of the four operating modes can be written using the low ripple approximation method as follows:

(A) Powering mode: During the $k-t h$ resonant interval, the output voltage is assumed to be nearly constant: that is, $v_{o}(t)=v_{o}(k)$. Since the inductor current and the capacitor voltage change sinusoidally, the $k-t h$ capacitor voltage and output voltage can be evaluated as follows:

$$
\begin{gathered}
v_{c}^{*}(k+1)=\frac{1}{C Z} \int_{0}^{T / 2}\left[v_{c}^{*}(k)+V_{s}-v_{o}(k)\right] \sin \left(\omega_{r} t\right) d t-v_{c}^{*}(k) \\
v_{o}(k+1)=\frac{1}{C_{o} Z} \int_{0}^{T / 2}\left[v_{c}^{*}(k)+\left\{V_{s}-v_{o}(k)\right\}\right] \sin \left(\omega_{r} t\right) d t \\
+v_{o}^{*}(k)-\frac{1}{C_{o}} \int_{0}^{T / 2} \frac{v_{o}(t)}{R} d t
\end{gathered}
$$

where, $T=2 \pi \sqrt{L C}$.

From (6) and (7), the state equation during the powering mode can be given as

$$
\left[\begin{array}{l}
v_{c}^{*}(k+1) \\
v_{o}(k+1)
\end{array}\right]=\left[\begin{array}{cc}
1 & -2 \\
\delta & 1-\delta-\delta^{*}
\end{array}\right]\left[\begin{array}{l}
v_{c}^{*}(k) \\
v_{o}(k)
\end{array}\right]+\left[\begin{array}{l}
2 \\
\delta
\end{array}\right]^{V_{s}}
$$

where,

$$
\begin{array}{r}
\delta=2 \frac{C}{C_{o}}, \delta^{*}=\frac{\pi}{2} Q \delta, \\
i_{p}^{*}(k)=\frac{v_{c}^{*}(k)+V_{s}-v_{o}(k)}{Z}
\end{array}
$$

(B) Free resonant mode: In this mode, all conditions are equal to those of the powering mode except $V_{s}$ being set to zero. Therefore, we can easily obtain the following state equation from (8) and (9)

$$
\begin{gathered}
{\left[\begin{array}{c}
v_{c}^{*}(k+1) \\
v_{o}(k+1)
\end{array}\right]=\left[\begin{array}{cc}
1 & -2 \\
\delta & 1-\delta-\delta^{*}
\end{array}\right]\left[\begin{array}{l}
v_{c}^{*}(k) \\
v_{o}(k)
\end{array}\right]} \\
i_{p}^{*}(k)=\frac{v_{c}^{*}(k)-v_{o}(k)}{Z}
\end{gathered}
$$

(C) Regeneration mode: In this mode, all of the conditions are equal to those of the powering mode except that $V_{s}$ is changed into $-V_{s}$. Therefore, we can obtain the following equations from (8) and (9) as: 

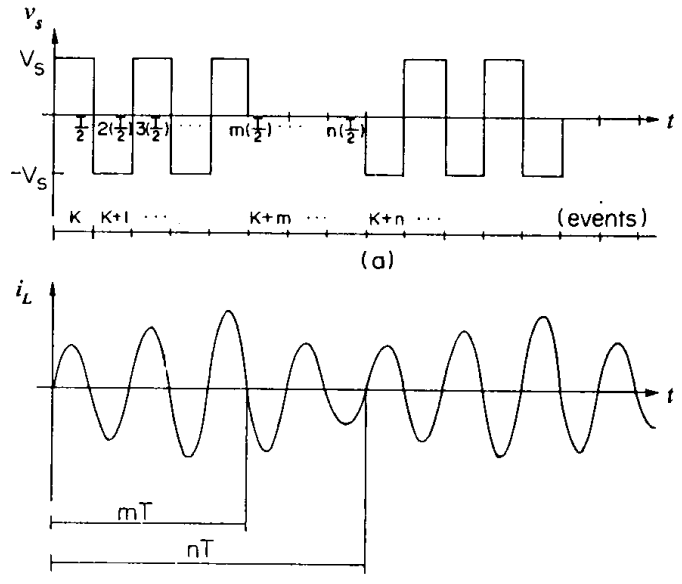

(b)

Fig. 7 Voltage $\left(v_{s}\right)$ and current $\left(i_{L}\right)$ waveforms of the tank circuit for duty ratio of $\mathrm{m} / \mathrm{n}$.

$$
\begin{aligned}
& {\left[\begin{array}{l}
v_{c}^{*}(k+1) \\
v_{o}(k+1)
\end{array}\right]=\left[\begin{array}{cc}
1 & -2 \\
\delta & 1-\delta-\delta^{*}
\end{array}\right]\left[\begin{array}{c}
v_{c}^{*}(k) \\
v_{o}(k)
\end{array}\right]+\left[\begin{array}{c}
-2 \\
-\delta
\end{array}\right]^{V_{s}}} \\
& i_{p}^{*}(k)=\frac{v_{c}^{*}(k)-v_{s}+v_{o}(k)}{Z} . \\
& \text { (D) Discontinuous mode: From (A4), and } \\
& v_{c}^{*}(k+1)=v_{c}^{*}(k) \text { in event } \mathrm{k} \text {, we obtain } \\
& {\left[\begin{array}{l}
v_{c}^{*}(k+1) \\
v_{o}(k+1)
\end{array}\right]=\left[\begin{array}{cc}
1 & 0 \\
0 & 1-\delta^{*}
\end{array}\right]\left[\begin{array}{l}
v_{c}^{*}(k) \\
v_{o}(k)
\end{array}\right]} \\
& i_{p}^{*}(k)=0 \quad .
\end{aligned}
$$

and

\section{A. DC transfer function}

Assume that the interval length of the powering mode is $m(T / 2)$ out of the total period $n(T / 2)$ as shown in Fig. 7, the dc transfer function can be given by a function of $m$ and $n$. If the new variables are defined as

$$
\begin{aligned}
& x_{1}(k)=v_{c}^{*}(k) \\
& x_{2}(k)=v_{o}(k) \\
& \mathbf{x}(k)=\left[\begin{array}{l}
x_{1}(k) \\
x_{2}(k)
\end{array}\right]
\end{aligned}
$$

and if the converter is operated in continuous mode, then the $m-t h$ state during the powering mode are found from (8), as follows:

$$
\begin{gathered}
\mathbf{x}(k+1)=\mathbf{A x}(k)+\mathbf{B} V_{s} \\
\mathbf{x}(k+2)=\mathbf{A}^{2} \mathbf{x}(k)+(\mathbf{A B}+\mathbf{B}) V_{s}
\end{gathered}
$$

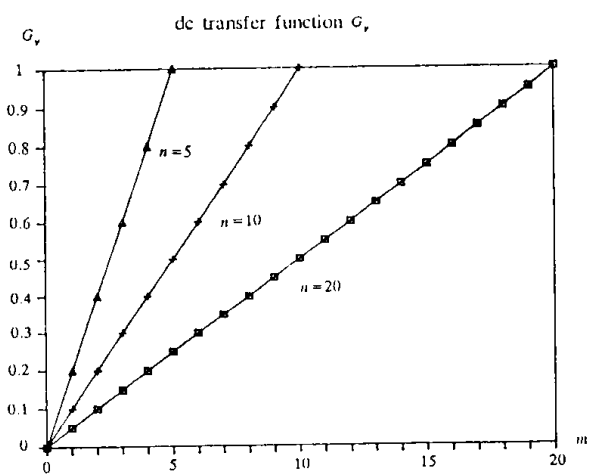

Fig. 8 Dc transfer function of the continuous resonant current switch modes.

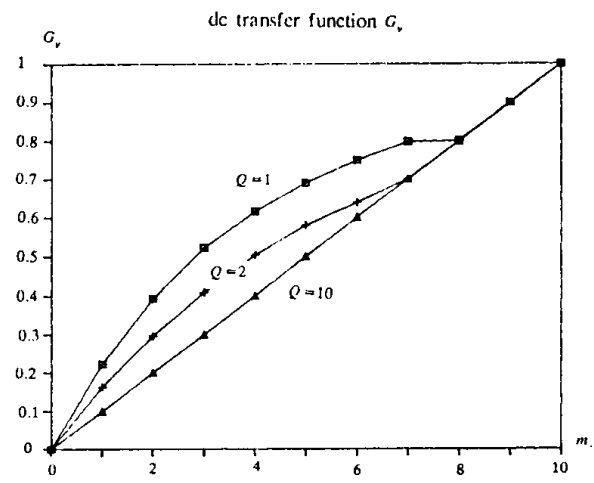

(a)

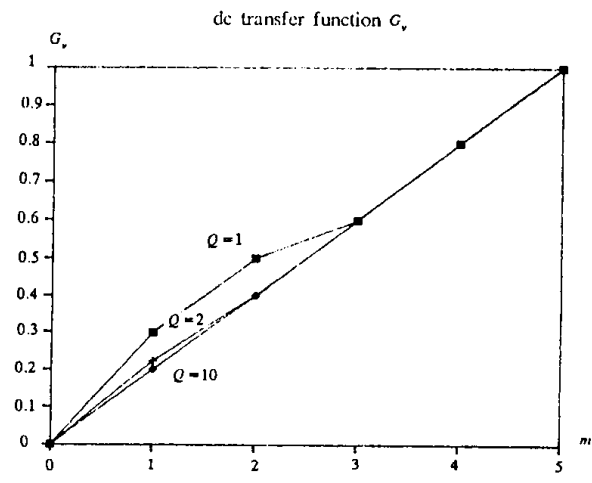

(b)

Fig. 9 Dc transfer function for different $Q$ and $n$ values: (a) $n=10$ (b) $n=5$

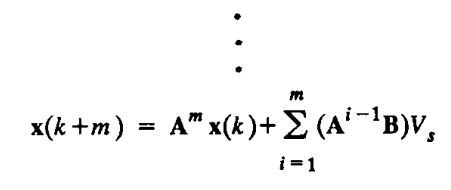

where,

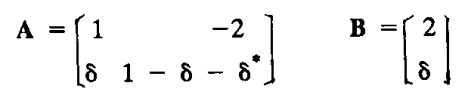




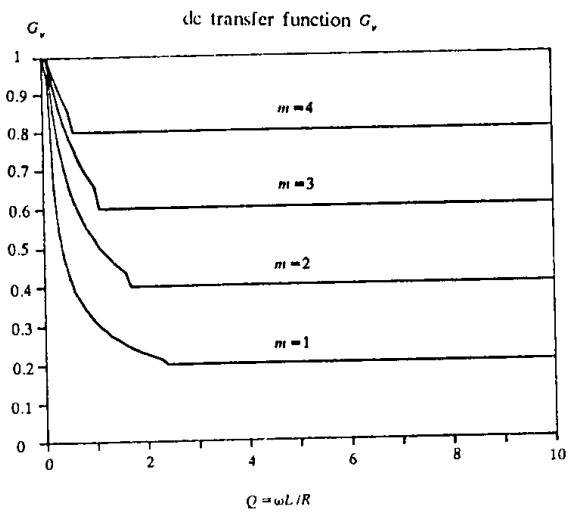

Fig. $10 \mathrm{Dc}$ transfer function as a function of load and $\mathrm{m}$ for $n=5$.

During the free resonant mode, the state equations can be evaluated by (10) as

$$
\mathbf{x}(k+n)=\mathbf{A}^{n-m} \mathbf{x}(k+m) .
$$

From (17) and (19), we obtain

$$
\mathbf{x}(k+n)=\mathbf{A}^{n} \mathbf{x}(k)+\mathbf{A}^{n-m} \sum_{i=1}^{m}\left(\mathbf{A}^{i-1} \mathbf{B}\right) V_{s} .
$$

In the steady state, it is reasonable to assume that

$$
\mathbf{x}(k)=\mathbf{x}(k+n) \text {. }
$$

Therefore $\mathbf{x}(k)$ is determined as follows:

$$
\mathbf{x}(k)=\left(\mathbf{I}-\mathbf{A}^{n}\right)^{-1} \mathbf{A}^{n-m} \sum_{i=1}^{m}\left(\mathbf{A}^{i-1} \mathbf{B}\right) V_{s}
$$

where, $I$ is identity matrix. Finally, the transfer function $G_{v}$ is given by

$$
G_{v}=v_{o} / V_{s}=C \mathbf{x}(k)=\mathbf{C}\left(\mathbf{I}-\mathbf{A}^{n}\right)^{-1}\left(\mathbf{A}^{n-m}\right) \sum_{i=1}^{m}\left(\mathbf{A}^{i-1} \mathbf{B}\right) .
$$

From (23), the transfer function $G_{v}$ is found to be dependent on $Q, C / C_{o}, m$ and $n$. If $Q \gg 1$, we see from Fig. 8 that the output voltage is directly proportional to the duty ratio $m / n$ . Note that the output voltage can be controlled in a wide range from zero to the maximum. However the output voltage level is discrete and the number of discrete levels are $n$. To control the output voltage by $\mathrm{m}$, the unit step voltage should be given by $V_{s} / n$. Thus in order to reduce the incremental step of the output voltage, the number of $n$ should be increased.

Fig. 9 shows the transfer function for different values of $Q$ and $n$. We can see from this curves, the dc transfer function deviates from the straight line as $Q$ and $m$ decreases and $n$ increases. This is because of the discontinuous mode. When $Q$ is small, the $L C$ tank energy also becomes small and the tank current fastly reduces to zero and it becomes discontinuous mode. When the duty ratio $m / n$ is small, the energy dissipation interval of $L C$ tank becomes longer than the energy storing interval and thus the discontinuous mode appears. When the discontinuous mode appears, the dc transfer function curve becomes nonlinear. In practice, however, the nonlinearity can be eliminated by feedback control.

Fig. 10 shows the dc transfer function as a function of load $R$ for three selected values of $m$ for $n=5$. We can see that the output voltage does not depend on the load as far as $R$ is smaller than a certain value. As $R$ becomes larger than the critical value, the output voltage becomes high because the discontinuous mode appears. From this figure, the $Q$ range can be determined for a given linear region if the load and the output voltage are given.

\section{B. Time Domain Simulation for feedback control scheme}

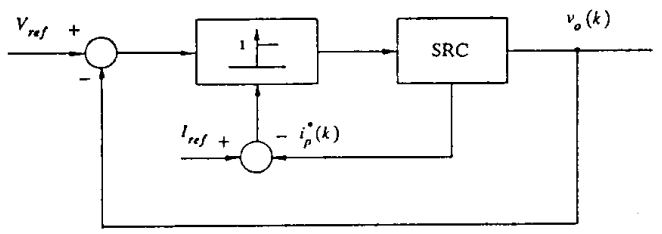

Fig. 11 The block diagram of the output voltage feedback control scheme.

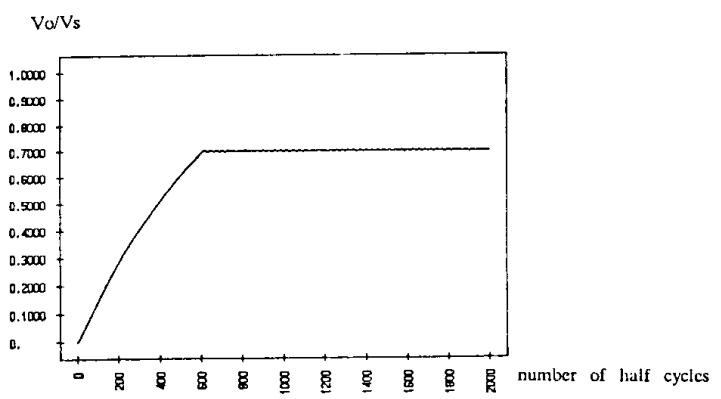

Fig. 12 Computer simulation result of a transient response. Normalized dc output voltage waveforms $\left(G_{v}\right)$ :

Conditions: $Z=200 \Omega, \quad C / C_{o}=0.0001, \quad Z / R=5, \quad I_{\text {ref }}=10 \mathrm{~A}$, $V_{o} / V_{\text {ref }}=0.7$

where $V_{\text {ref }}=$ reference output voltage and $I_{\text {ref }}=$ reference inductor peak current.

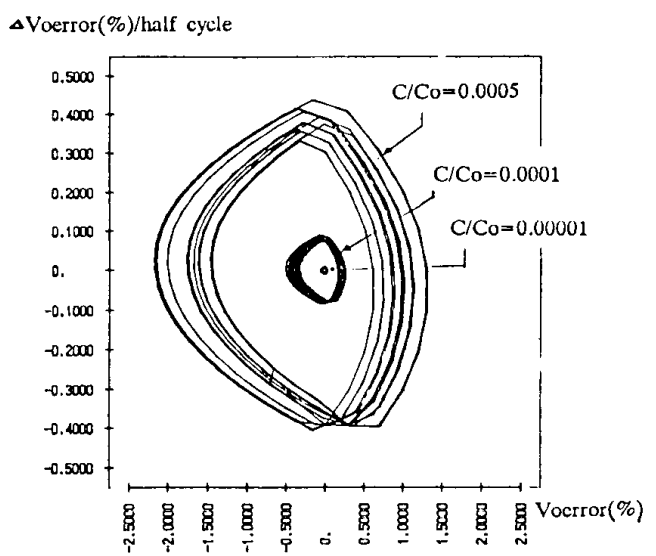

Fig. 13 Diagram of the steady state limit cycle on the phase plane for the output error voltage $V_{o \text { error }}$ where $V_{\text {ofrror }}=\left(\left(V_{0}-V_{\text {ref }}\right) / V_{\text {ref }}\right) \times 100$. 
Fig. 11 shows the block diagram which is used for the experiment. In this figure, the duty ratio of the powering mode and the free resonant mode with (or without) discontinuous mode is controlled by the time domain selection of the switch modes from the difference signal between the output voltage and the command voltage. Therefore the output voltage can be effectively regulated by feedback control. In the miner loop, the peak resonant current is limited to a predetermined value to protect the power device during the powering mode operation. Especially, in the transient or load dynamic state, the resonant current can be effectively limited to the predetermined value.

Fig. 12 shows the simulation result of the control diagram when the output voltage command is given as a unit step function. The output voltage has no overshoot during the transient state and it is regulated during the steady state. The transient response time is a function of filter size, load condition and $I_{\text {ref }}$

One demerit of such a kind of control scheme is the output voltage ripple during the steady state operation due to the limit cycle phenomenon[10]. Fig. 13 shows the diagram of the steady state limit cycle on phase plane, where the magnitude depends on the capacitance ratio of $C$ and $C_{o}$. Of course, such a kind of limit cycle can be reduced further by a proper feedback control or by inserting an additional filter at the output side.

\section{EXPERIMENTS}

In the experiment, the full bridge converter shown in Fig. 1 is used. The converter is designed to have the maximum output power $P_{\max }=250 \mathrm{~W}$ at $100 \mathrm{KHz}$ of operating frequency. The parameter values of the converter are given as follows:

$$
\begin{aligned}
& L=258 \mu H \\
& C=0.0106 \mu F \\
& Z=256 \Omega \\
& C_{o}=470 \mu \mathrm{F}
\end{aligned}
$$

The voltage and current waveforms flowing on switch S2 are shown in Fig. 14. This oscillogram shows that the switching stress is low because the switching instants are synchronized to the current zero crossing points.

The waveforms of the resonant current and switch voltage are shown in Fig. 15 where the duty ratio are 1/10, 2/10, $\ldots, 9 / 10$ and $10 / 10$, respectively. In these oscillograms, we can observe that the powering and free resonant modes are repeated alternately and the switching instants are always synchronized to the current zero crossing points.

Fig. 16 shows the comparison of the measured data and the theoretical values for $n=10$ and $Q=1(R=156 \Omega)$ $Q=2(R=78 \Omega)$ and $Q=5(R=31.2 \Omega)$, respectively. When $Q=5$, the theoretical curve with conduction loss compensation has lower gain than the ideal one shown in Fig. 16(c) because of the FET series resistance $(0.3 \Omega)$, inductor series resistance $(0.88 \Omega)$, capacitor series resistance $(0.79 \Omega)$ and rectified diode voltage drop $0.6 \mathrm{~V}$. The transformer is eliminated in this experiment. The measured values are nearly coincided with the theoretical values when the conduction losses are considered In the light $\operatorname{load}(\mathrm{Q}=1$ and $\mathrm{Q}=2)$ condition, the conduction loss term is small and the measured value are nearly coincided with the theoretical ones as shown in Fig. 16(a) and (b).

Fig. 17 shows an experimental result of the transient response when the output voltage command is given by a unit step function. The result shows good regulation with no overshoot.

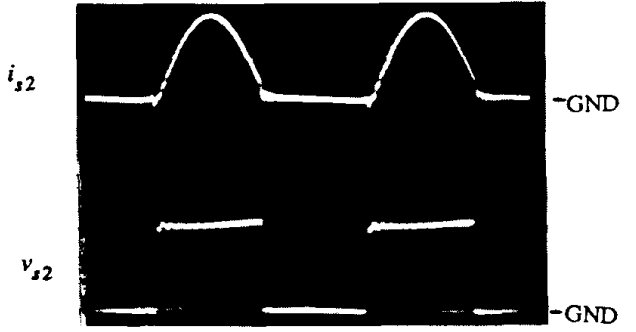

Fig. 14 Waveforms of the voltage $v_{s 2}$ and current $i_{s 2}$ for switch S2 $v_{s 2}: 50 \mathrm{~V} / \mathrm{div}, i_{s 2}: 2 \mathrm{~A} / \mathrm{div}$. time:2 $\mu \mathrm{sec} / \mathrm{div}$.
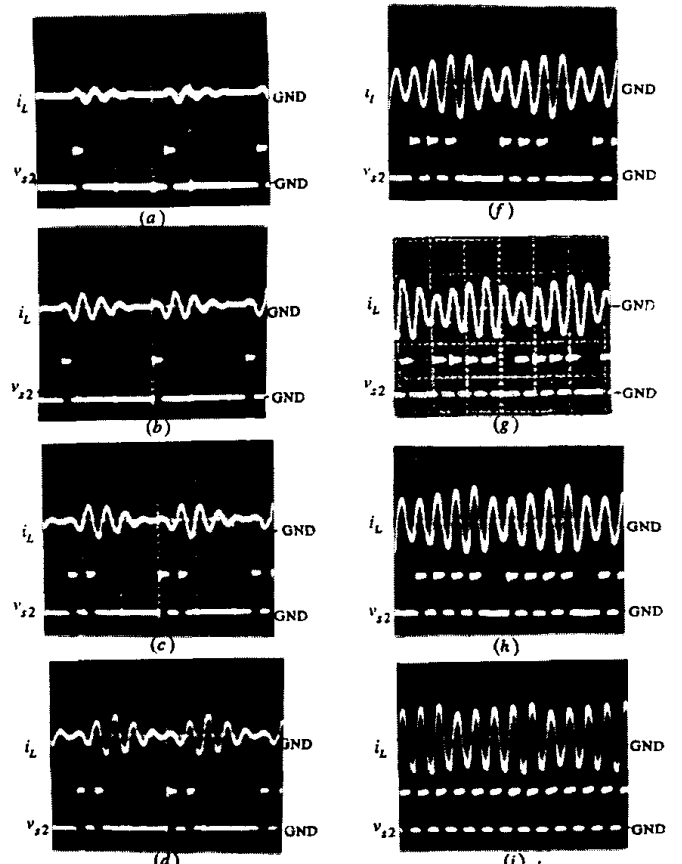

(k)
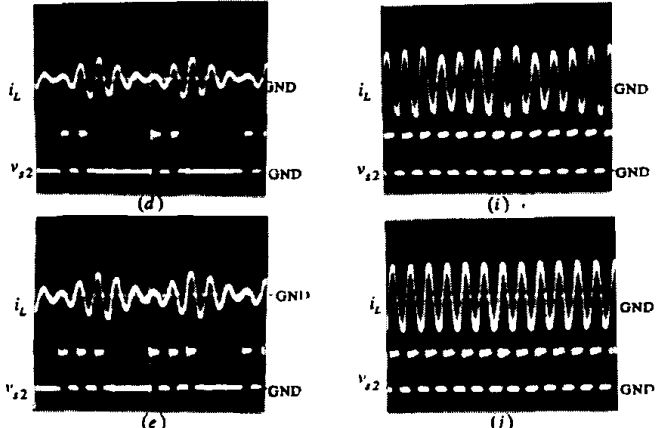

(e)

Fig. 15 The waveforms of the switch in duty ratio: (a) 1/10 (b) $2 / 10$ (c) $3 / 10$ (d) $4 / 10$ (e) $5 / 10$ (f) $6 / 10$ (g) $7 / 10$ (b) $8 / 10$ (i) $9 / 10$ (j) $10 / 10$. Inductor current $i_{L}: 5 \mathrm{~A} / \mathrm{div}$, switch voltage $v_{s 2}: 100 \mathrm{~V} / \mathrm{div}$, time : $20 \mu \mathrm{sec} / \mathrm{div}$.

\section{CONCLUSION}

A new control scheme of the SRC is suggested in this paper. The SRC operates at the circuit resonant frequency and the switching instants are always synchronized to the current zero crossing points. Therefore the device loss is only conduc- 


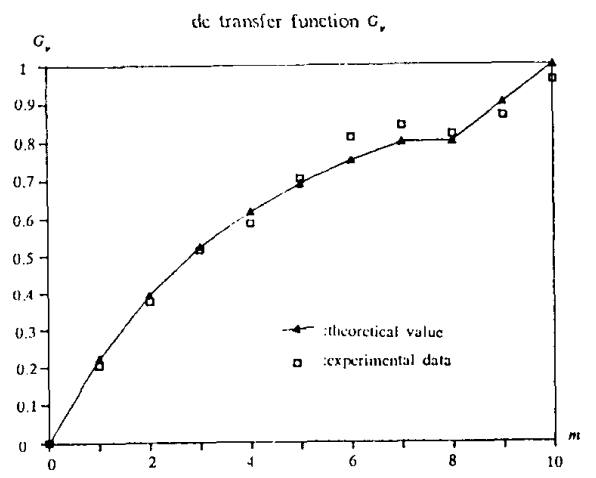

(a)

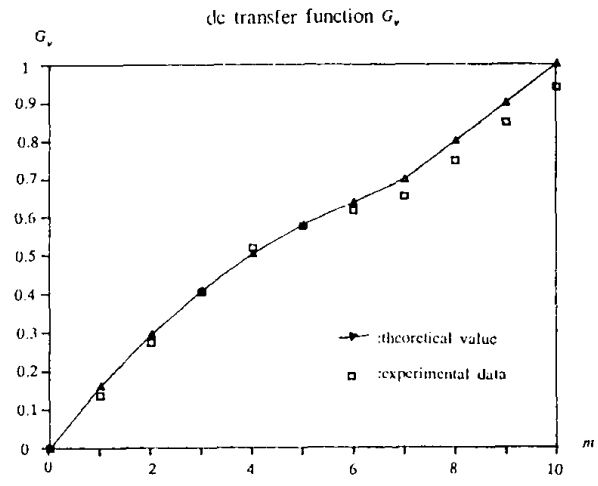

(b)

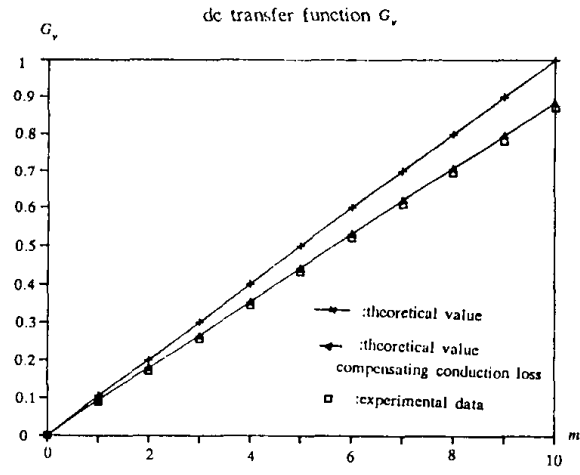

(c)

Fig. 16 The comparison of the transfer function between theoretical and experimental results for (a) $Q=1(R=156 \Omega)$ (b) $Q=2(R=78 \Omega)(\mathrm{c}) Q=5(\mathrm{R}=31.2 \Omega)$.

tion loss and the device capability is fully utilized under high frequency operation. Converter switching frequency is not limited by the switching loss but by the device turn on/off time The output voltage control is achieved by varying the interval length ratio of the switch modes. This control scheme is analyzed in detail and the characteristics are verified through the experiment. The properties of the proposed scheme are summarized as follows:

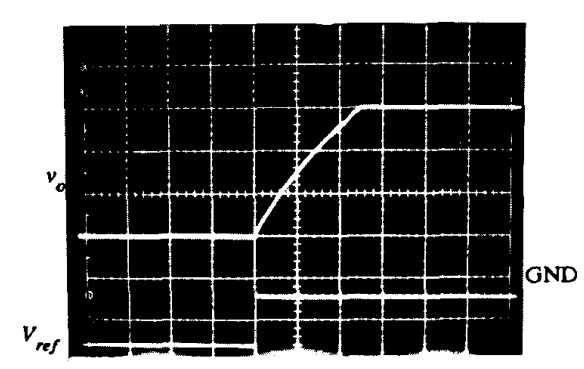

Fig. 17 Transient response of the output voltage $v_{0}$ for command voltage $V_{\text {ref }}: v_{o}: 20 \mathrm{~V} / \mathrm{div}$, time: $2 \mathrm{msec} / \mathrm{div}$.

(1) SRC always guarantees many advantages such as zero device switching current, low switching loss, reliable high frequency operation and low EMI because SRC always operates at optimal condition.

(2) For high $Q$, the dc transfer function is linear by the duty ratio of the powering mode and free resonant mode and it is independent of the load. Despite of light load condition, the output voltage can be controlled from zero to the maximum.

(3) Two demerits of this scheme are discrete output voltage levels and the output voltage ripple due to the limit cycle phenomenon. However the output voltage can be controlled by feedback scheme and the output voltage ripple can be reduced further by a good feedback control scheme or by inserting an additional filter at the output side.

\section{REFERENCES}

[1] Sjored W. H. De Hann, "A new integral pulse module for the series-resonant converter," IEEE Trans. Ind. Electronics, Vol. IE-31, No. 3, pp. 255-262, Aug. 1984

[2] Robert L. Steigerward, "High frequency resonan ttransistor dc-dc converters," IEEE Trans. Ind. Electronics. Vol. IE-31, No. 2, pp.181-192, May 1984.

[3] F. C. Schwarz, "An improved method of resonant current pulse modulation for power converters," IEEE. Trans. Ind. Electronics. Contro. Intrum., Vol. IECI-23, No. 2, pp.133141, May 1976.

[4] R. J. King and T. A. Sturat, "A large signal dynamic simulation for the series resonant converter," IEEE Trans. on Aerospace and Electronic Systems. Vol. AES-19. No. 6, pp. 859-870, Nov. 1983.

[5] R. J. King and T. A. Sturat, "Inherent overload protection for the series resonant converter," IEEE. Trans. on. Aerospace and Electronic Systems. Vol. AES-19. No. 6, pp. 820-830, Nov. 1983.

[6] D. M. Divan, "Design considerations for very high frequency resonant mode dc/dc converters," Conference record of the of the 1986 IEEE Industry Application Society Annual Meeting pp. 640-647.

[7] Vatche Vorperian and Slobodan Cuk, "Small signal analysis of resonant converters," IEEE PESC Rec., 269-282, Jun. 1983.

[8] A. S. Kislovski, "A contribution for steady-state modeling of half-bridge series-resonant power cells," IEEE. Trans. on Power Electronics, Vol. PE-1, No. 3, July 1986. 
[9] Ira J. Pitel, "Phase-modulated resonant power conversion techniques for high frequency link inverters," IEEE Trans. Ind. Appl. vol. IA-22, no. 6, Nov. 1986.

[10] Konstanty J. Kurman, "Feedback control theory and design"

\section{Appendix I.}

From Fig. 3, the differential equations of the system for each switch mode drived as follows:

Powering mode:

$\left[\begin{array}{l}\dot{v}_{c}^{*}(t) \\ i_{L}^{*}(t) \\ \dot{v}_{o}(t)\end{array}\right]=\left[\begin{array}{ccc}0 & \frac{1}{C Z} & 0 \\ -\frac{1}{L} & 0 & -\frac{1}{L} \\ 0 & \frac{1}{C_{o} Z} & \frac{-1}{C_{o} R}\end{array}\right]\left[\begin{array}{c}v_{c}^{*}(t) \\ i_{L}^{*}(t) \\ v_{o}(t)\end{array}\right]+\left[\begin{array}{c}0 \\ \frac{1}{L} \\ 0\end{array}\right] v_{s}$

where, $v_{c}^{*}(t)=v_{c}(t), \quad i_{L}^{*}(t)=i_{L}(t)$ for Q1,Q4 on.

$v_{c}^{*}(t)=-v_{c}(t), \quad i_{L}^{*}(t)=-i_{L}(t)$ for Q2,Q3 on.

Free resonant mode:

$$
\left[\begin{array}{l}
\dot{v}_{c}^{*}(t) \\
i_{L}^{*}(t) \\
\dot{v}_{o}(t)
\end{array}\right]=\left[\begin{array}{ccc}
0 & \frac{1}{C Z} & 0 \\
-\frac{1}{L} & 0 & -\frac{1}{L} \\
0 & \frac{1}{C_{o} Z} & \frac{-1}{C_{o} R}
\end{array}\right]\left[\begin{array}{c}
v_{c}^{*}(t) \\
i_{L}^{*}(t) \\
v_{o}(t)
\end{array}\right]
$$

where, $v^{*}(t)=v_{c}(t), \quad i^{*}(t)=i_{L}(t)$ for $\mathrm{Q} 4, \mathrm{D} 2$ on. $v_{c}^{*}(t)=-v_{c}(t), \quad i_{L}^{*}(t)=-i_{L}(t)$ for Q2,D4 on.

Regeneration mode:

$$
\left[\begin{array}{c}
\dot{v}_{c}^{*}(t) \\
i_{L}^{*}(t) \\
\dot{v}_{o}(t)
\end{array}\right]=\left[\begin{array}{ccc}
0 & \frac{1}{C Z} & 0 \\
-\frac{1}{L} & 0 & \frac{1}{L} \\
0 & \frac{1}{C_{o} Z} & \frac{-1}{C_{o} R}
\end{array}\right]\left[\begin{array}{c}
v_{c}^{*}(t) \\
i_{L}^{*}(t) \\
v_{o}(t)
\end{array}\right]+\left[\begin{array}{c}
0 \\
\frac{-1}{L} \\
0
\end{array}\right] v_{s}^{v_{s}}
$$

where, $v_{c}^{*}(t)=v_{c}(t), i_{L}^{*}(t)=i_{L}(t)$ for $\mathrm{D} 2, \mathrm{D} 3$ on. $v_{c}^{*}(t)=-v_{c}^{c}(t), \quad i_{L}^{*}(t)=-i_{L}(t)$ for $\mathrm{D} 1, \mathrm{D} 4$ on.

Discontinuous mode:

$$
\left[\begin{array}{l}
\dot{v}_{c}^{*}(t) \\
\dot{i}_{L}^{*}(t) \\
\dot{v}_{o}(t)
\end{array}\right]=\left[\begin{array}{ccc}
0 & 0 & 0 \\
0 & 0 & 0 \\
0 & 0 & \frac{-1}{C_{0} R}
\end{array}\right]\left[\begin{array}{l}
v_{c}^{*}(t) \\
i_{L}^{*}(t) \\
v_{o}(t)
\end{array}\right]
$$

where, $v_{c}^{*}(t)=v_{c}(t), \quad i_{L}^{*}(t)=i_{L}(t)$ 